AIP Applied Physics

\title{
Analysis of the modified optical properties and band structure of GaAs1-xSbx-capped InAs/GaAs quantum dots
}

J. M. Ulloa, J. M. Llorens, M. del Moral, M. Bozkurt, P. M. Koenraad et al.

Citation: J. Appl. Phys. 112, 074311 (2012); doi: 10.1063/1.4755794

View online: http://dx.doi.org/10.1063/1.4755794

View Table of Contents: http://jap.aip.org/resource/1/JAPIAU/v112/i7

Published by the American Institute of Physics.

\section{Related Articles}

Pronounced Purcell enhancement of spontaneous emission in CdTe/ZnTe quantum dots embedded in micropillar cavities

Appl. Phys. Lett. 101, 132105 (2012)

Fabrication and photoluminescence of $\mathrm{SiC}$ quantum dots stemming from $3 \mathrm{C}, 6 \mathrm{H}$, and $4 \mathrm{H}$ polytypes of bulk $\mathrm{SiC}$ Appl. Phys. Lett. 101, 131906 (2012)

Radiative transitions in stacked type-II ZnMgTe quantum dots embedded in $\mathrm{ZnSe}$

J. Appl. Phys. 112, 063521 (2012)

Anomalous temperature dependence of photoluminescence in self-assembled InGaN quantum dots Appl. Phys. Lett. 101, 131101 (2012)

Optical properties of multi-stacked InGaAs/GaNAs quantum dot solar cell fabricated on GaAs (311)B substrate J. Appl. Phys. 112, 064314 (2012)

\section{Additional information on J. Appl. Phys.}

Journal Homepage: http://jap.aip.org/

Journal Information: http://jap.aip.org/about/about_the_journal

Top downloads: http://jap.aip.org/features/most_downloaded

Information for Authors: http://jap.aip.org/authors

\section{ADVERTISEMENT}

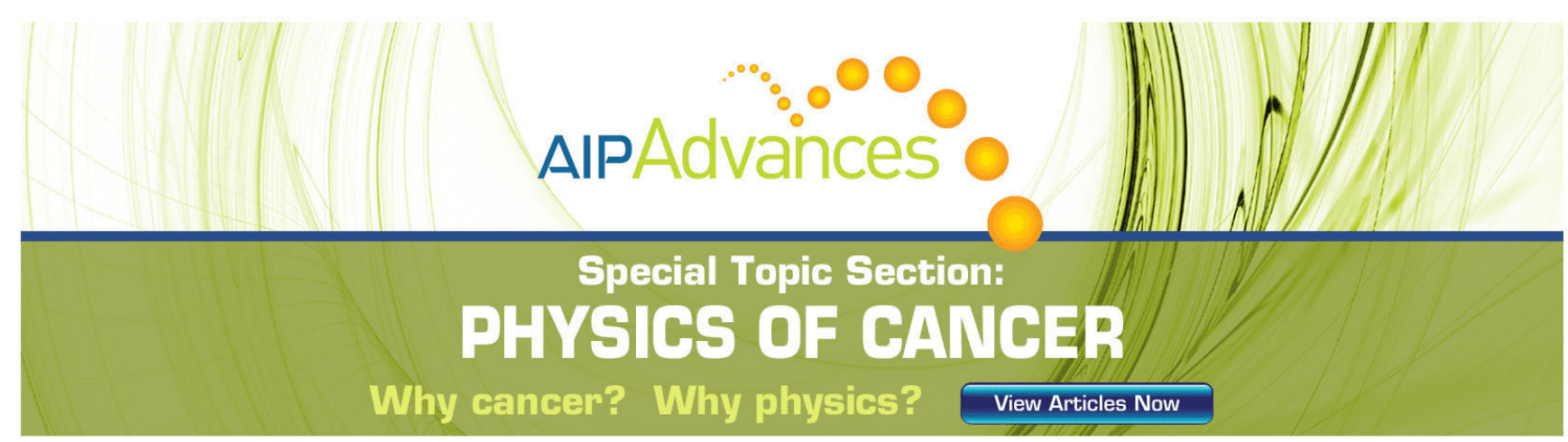




\title{
Analysis of the modified optical properties and band structure of $\mathrm{GaAs}_{1-\mathrm{x}} \mathrm{Sb}_{\mathrm{x}}$-capped InAs/GaAs quantum dots
}

\author{
J. M. Ulloa, ${ }^{1, a)}$ J. M. Llorens, ${ }^{2}$ M. del Moral, ${ }^{1}$ M. Bozkurt, ${ }^{3}$ P. M. Koenraad, ${ }^{3}$ and A. Hierro ${ }^{1}$ \\ ${ }_{1}^{1}$ Institute for Systems based on Optoelectronics and Microtechnology (ISOM), Universidad Politecnica de \\ Madrid, Ciudad Universitaria s/n, 28040 Madrid, Spain \\ ${ }^{2}$ IMM-Instituto de Microelectrónica de Madrid (CNM-CSIC), Isaac Newton 8, PTM, E-28760 Tres Cantos, \\ Madrid, Spain \\ ${ }^{3}$ Photonics and Semiconductor Nanophysics, Department of Applied Physics, Eindhoven University \\ of Technology, P. O. Box 513, NL-5600 MB Eindhoven, The Netherlands
}

(Received 25 July 2012; accepted 29 August 2012; published online 4 October 2012)

The origin of the modified optical properties of InAs/GaAs quantum dots (QD) capped with a thin $\mathrm{GaAs}_{1-\mathrm{x}} \mathrm{Sb}_{\mathrm{x}}$ layer is analyzed in terms of the band structure. To do so, the size, shape, and composition of the QDs and capping layer are determined through cross-sectional scanning tunnelling microscopy and used as input parameters in an $8 \times 8 \mathrm{k} \cdot \mathrm{p}$ model. As the $\mathrm{Sb}$ content is increased, there are two competing effects determining carrier confinement and the oscillator strength: the increased QD height and reduced strain on one side and the reduced QD-capping layer valence band offset on the other. Nevertheless, the observed evolution of the photoluminescence (PL) intensity with $\mathrm{Sb}$ cannot be explained in terms of the oscillator strength between ground states, which decreases dramatically for $\mathrm{Sb}>16 \%$, where the band alignment becomes type II with the hole wavefunction localized outside the QD in the capping layer. Contrary to this behaviour, the PL intensity in the type II QDs is similar (at $15 \mathrm{~K}$ ) or even larger (at room temperature) than in the type I Sb-free reference QDs. This indicates that the PL efficiency is dominated by carrier dynamics, which is altered by the presence of the GaAsSb capping layer. In particular, the presence of Sb leads to an enhanced PL thermal stability. From the comparison between the activation energies for thermal quenching of the PL and the modelled band structure, the main carrier escape mechanisms are suggested. In standard GaAs-capped QDs, escape of both electrons and holes to the GaAs barrier is the main PL quenching mechanism. For small-moderate $\mathrm{Sb}(<16 \%)$ for which the type I band alignment is kept, electrons escape to the GaAs barrier and holes escape to the $\mathrm{GaAsSb}$ capping layer, where redistribution and retraping processes can take place. For $\mathrm{Sb}$ contents above $16 \%$ (type-II region), holes remain in the GaAsSb layer and the escape of electrons from the QD to the GaAs barrier is most likely the dominant PL quenching mechanism. This means that electrons and holes behave dynamically as uncorrelated pairs in both the type-I and type-II structures. @ 2012 American Institute of Physics. [http://dx.doi.org/10.1063/1.4755794]

\section{INTRODUCTION}

Since the use of $\mathrm{Sb}$ to cover InAs/GaAs quantum dots (QDs) was first reported a few years ago, ${ }^{1-3}$ significant activity has been carried out in this field, which is becoming increasingly attractive. ${ }^{4-22}$ The success of this approach to modify the optical and structural properties of InAs/GaAs QDs has led to the extrapolation of Sb-containing capping layers to InAs QDs grown on InP substrates, ${ }^{23}$ thereby broadening the field of applications of GaAsSb capping layers. A thin $(\sim 5 \mathrm{~nm})$ GaAsSb capping layer allows extending the emission wavelength of InAs QDs grown on GaAs substrates to the $1.55 \mu \mathrm{m}$ region. ${ }^{1-5}$ Additionally, the room temperature photoluminescence (PL) of the InAs QDs has been shown to be improved (increased integrated intensity and reduced inhomogeneous broadening) by the GaAsSb capping layer with moderate $\mathrm{Sb}$ contents. ${ }^{18}$ This improvement has been attributed to an increased QD height due to reduced In-Ga intermixing during the capping process, ${ }^{18}$ which would increase carrier localization and electron-hole

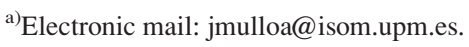

wavefunction overlap. Although a progressive increase in the QD height with the Sb content up to $\sim 14 \%$ has been demonstrated, it is not clear whether this effect can dominate over the counteracting action of the reduced QD-capping layer valence band offset with $\mathrm{Sb}$, which tends to delocalize the hole wavefunction towards the capping layer and reduce the overlap with the electron wavefunction. In addition, the impact of Sb on the PL properties could be strongly temperature dependent. The increase in QD height, together with the reduced strain and the transition to a type-II band alignment at high $\mathrm{Sb}$ contents $(\sim 16 \%)$, should significantly affect the carrier escape mechanisms and the temperature evolution of the QD PL emission in these samples.

In this work, we study how the presence of $\mathrm{Sb}$ in the capping layer alters the PL properties of InAs/GaAs QDs and their evolution with temperature. We accurately determine the Sb content by extrapolation from the unique relationship found between the $\mathrm{Sb}_{4}$ flux used during growth, the real $\mathrm{Sb}$ content in the capping layer as measured by crosssectional scanning tunnelling microscopy (X-STM), and the PL peak wavelength in the same QD layers. We next study the evolution of the PL properties of the QDs with 

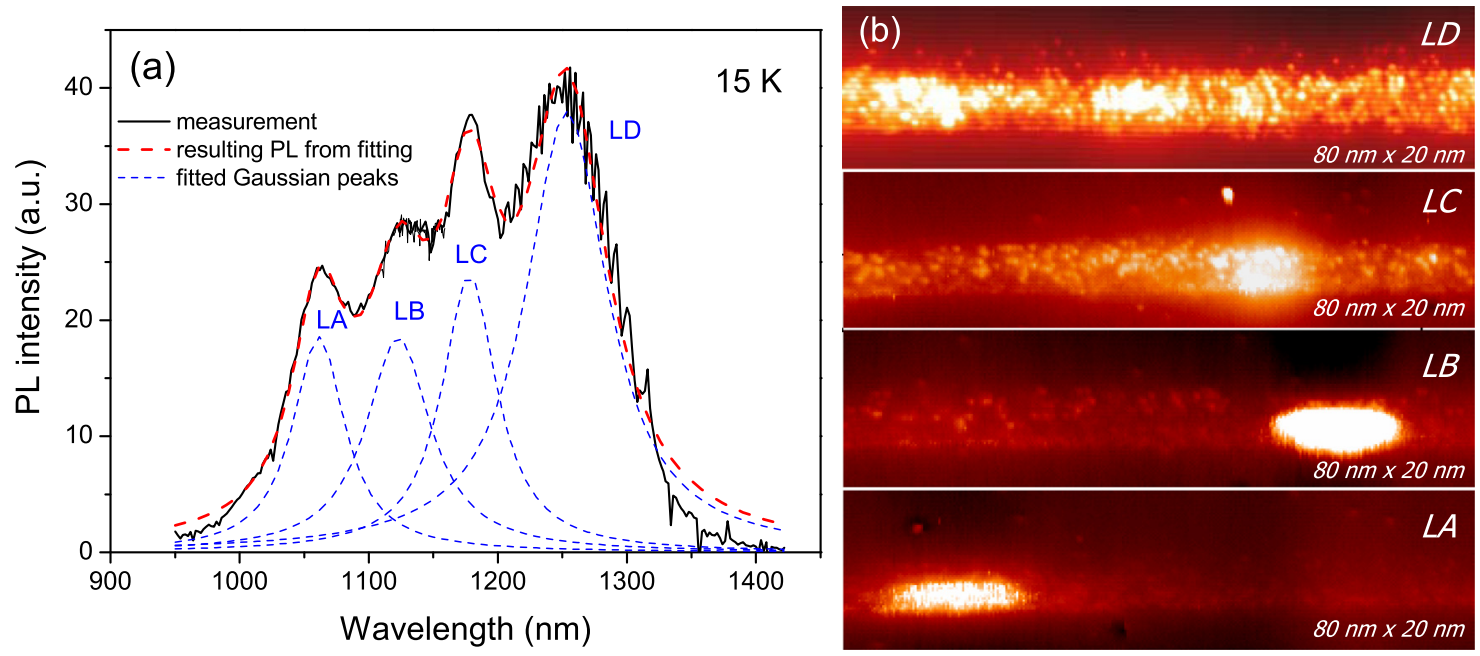

FIG. 1. (a) $15 \mathrm{~K}$ PL spectra of the sample with four QD layers designed for X-STM measurements. A four peak Gaussian fit to the measured data is also shown. (b) X-STM topography images $(\mathrm{V}=-3 \mathrm{~V})$ of the four different QD layers. The GaAsSb layer with higher amount of Sb appears brighter in the image. One QD can be observed in each of the first three layers.

temperature as a function of the $\mathrm{Sb}$ content. The experimental results are interpreted in terms of the band structure obtained with an $8 \times 8 \mathrm{k} \cdot \mathrm{p}$ method, in which a realistic structure with the size, shape, and composition of the QD and capping layer obtained from X-STM measurements is considered.

\section{EXPERIMENTAL PROCEDURE}

The samples were grown by solid source molecular beam epitaxy (MBE) on $\mathrm{n}^{+} \mathrm{Si}$ doped (100) GaAs substrates. A series of six samples containing a single QD layer were grown for PL studies. In all of these samples, 2.7 monolayers (MLs) of InAs were deposited at $450{ }^{\circ} \mathrm{C}$ and $0.04 \mathrm{ML} / \mathrm{s}$ on an intrinsic GaAs buffer layer and capped with a nominally $4.5 \mathrm{~nm}$-thick $\mathrm{GaAs}_{1-\mathrm{x}} \mathrm{Sb}_{\mathrm{x}}$ layer grown at $470{ }^{\circ} \mathrm{C}$. The $\mathrm{Sb}$ content was nominally changed from 0 to $30 \%$. A thick layer of $250 \mathrm{~nm}$ of GaAs was grown on top of the GaAsSb capping. Four of the GaAsSb-capped QD layers with different Sb contents were reproduced in a single sample (separated from each other by $50 \mathrm{~nm}$ of GaAs) for X-STM measurements.

The PL was measured from $15 \mathrm{~K}$ up to room temperature using a He-Ne laser as the excitation source, i.e., excitation takes place in the GaAs surrounding the nanostructures. The emitted light was dispersed through a $1 \mathrm{~m}$ spectrometer and detected with a liquid nitrogen cooled Ge detector. The X-STM measurements were performed on a (l 110$)$ surface plane of in-situ cleaved samples under ultra high vacuum $\left(p<4 \times 10^{-11}\right.$ Torr) conditions at room temperature. Polycrystalline tungsten tips prepared by electrochemical etching were used. The images were obtained in constant current mode at high negative voltages $(\sim 3 \mathrm{~V})$.

\section{RESULTS}

\section{A. Determination of the Sb content}

In order to determine the $\mathrm{Sb}$ content in the analyzed samples, a relationship between the $\mathrm{Sb}_{4}$ flux used during growth, the real Sb content measured by X-STM, and the PL peak wavelength was established by determining these three parameters in the same QD layers. An X-STM topography image of the four QD layers in the sample designed for structural characterization is shown in Fig. 1, together with the low temperature PL spectrum of the same sample. The $\mathrm{Sb}$ content in the capping layer was different in each layer (labeled LA, LB, LC, and LD) starting from $0 \%$ (reference GaAs-capped QDs) in the first layer (LA). In these images, the measured brightness of the layers is proportional to the amount of $\mathrm{Sb}$ mainly due to the increased compressive strain. The capping layer thickness was found to be $4.0 \pm 0.5 \mathrm{~nm}$ in all cases. The PL emission of the four layers can be clearly distinguished and the PL spectra can be reasonably well fitted by four Gaussian curves (Fig. 1(a)), each corresponding to the PL of one of the QD layers. From high negative voltage $(-3 \mathrm{~V}) \mathrm{X}-\mathrm{STM}$ images like those shown in Fig. 1(b), the Sb content in the capping layer can be deduced by analyzing the strain-induced outward relaxation of the cleaved surface. ${ }^{24,25}$ By comparing the measured outward relaxation to calculations from continuum elasticity theory, we obtain an Sb content of 0, 7, 11, and 22\% for LA, LB, LC, and LD, respectively. The size, shape, and composition of the QDs will be described in detail in Sec. III C.

By plotting the PL peak wavelength as a function of the $\mathrm{Sb}_{4}$ beam equivalent pressure (BEP) and the measured $\mathrm{Sb}$ content for the four layers (Fig. 2), we see that, to first order, the relationship can be considered linear. A univocal relationship between the three parameters is obtained, meaning that the incorporated amount of $\mathrm{Sb}$ is proportional to the $\mathrm{Sb}_{4}$ flux used during growth and this determines the PL peak wavelength. Since this relationship is unique for all the samples grown under the same conditions (same QDs and same thickness of the capping layer), the Sb content in the six single QD layer samples (from now on called A to F) can be obtained from the measured $\mathrm{Sb}_{4} \mathrm{BEP}$ (or the low temperature PL peak energy) by interpolation to this curve. The inset in Fig. 2 shows the deduced $\mathrm{Sb}$ content for samples $\mathrm{A}$ to $\mathrm{F}$, 


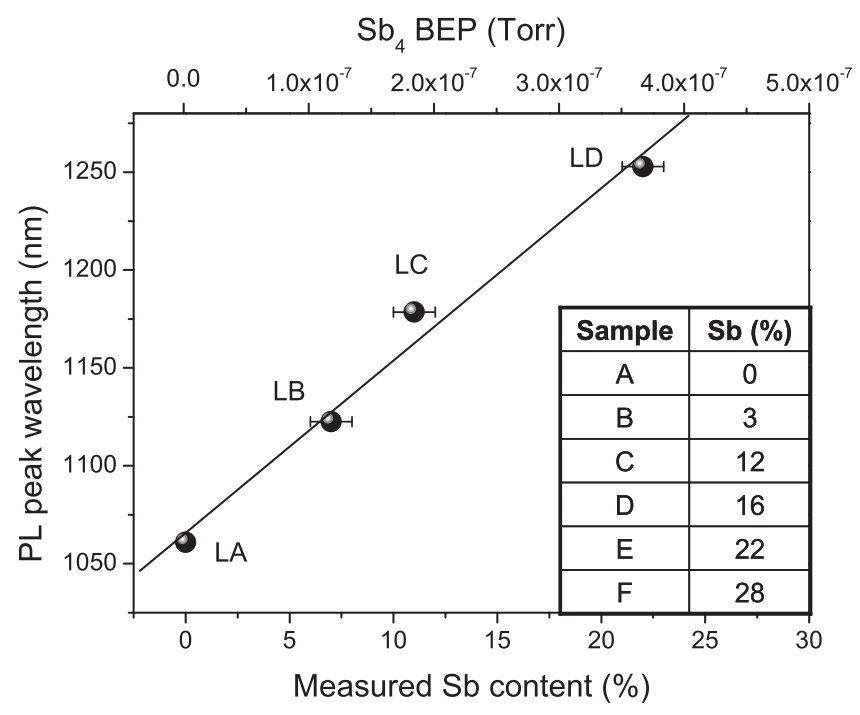

FIG. 2. $15 \mathrm{~K} \mathrm{PL}$ peak wavelength as a function of the Sb content measured by X-STM (bottom axis) and the $\mathrm{Sb}_{4}$ BEP used during growth (top axis) for the four layers of the sample shown in Fig. 1. Notice that both $\mathrm{x}$ axes are linear, meaning that the incorporated amount of $\mathrm{Sb}$ is proportional to the used $\mathrm{Sb}_{4}$ BEP. The line is a linear fit to the data. The inset shows the Sb content deduced for samples A to $\mathrm{F}$ from extrapolation to the linear fit.

for which the $15 \mathrm{~K}$ PL spectra are shown in Fig. 3. The Sb content of the sample emitting at the longest wavelength $\mathrm{F}$ $(28 \%)$ is, as far as we know, the highest reported.

\section{B. Optical properties}

Fig. 3 shows the $15 \mathrm{~K}$ PL spectra of the samples in the series with increasing amount of $\mathrm{Sb}$ in the capping layer (A to F). As already reported, ${ }^{1-5}$ by gradually increasing the $\mathrm{Sb}$ content, the emission wavelength of the QDs can be redshifted. The red shift is due to the reduction in strain, ${ }^{1-5}$ the increase in QD height, ${ }^{18}$ and the transition to a type-II band alignment in the valence band, with the hole wavefunction being localized out of the QD, in the GaAsSb capping layer. $^{3,4,13,14}$ Sample E is the first sample to show a clear

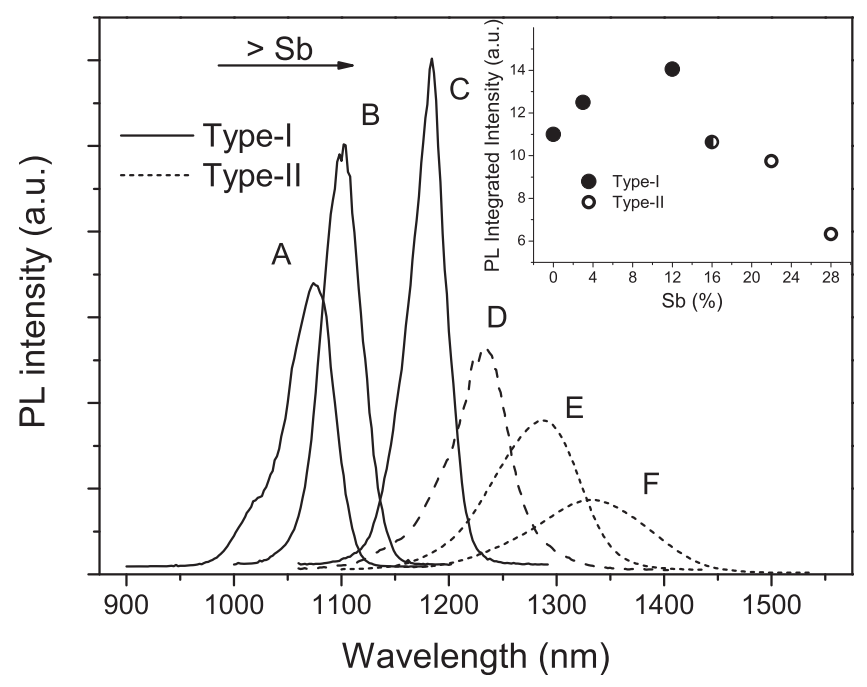

FIG. 3. $15 \mathrm{~K}$ PL spectra from the QD layers with increasing amount of Sb in the capping layer. The inset shows the PL integrated intensity as a function of the Sb content.
type-II band alignment behavior that is also present in all the QD layers emitting at longer wavelengths. This is evident from the observed blue shift of the PL peak energy with excitation power, which is not present in the shorter wavelength layers (Fig. 4). This blue shift is due to the band bending induced by the spatially separated photoexcited carriers in a type-II band alignment. ${ }^{10}$ Nevertheless, sample D already shows some signatures typical of the type-II band alignment (see Fig. 4(a)) which are not present in the lower
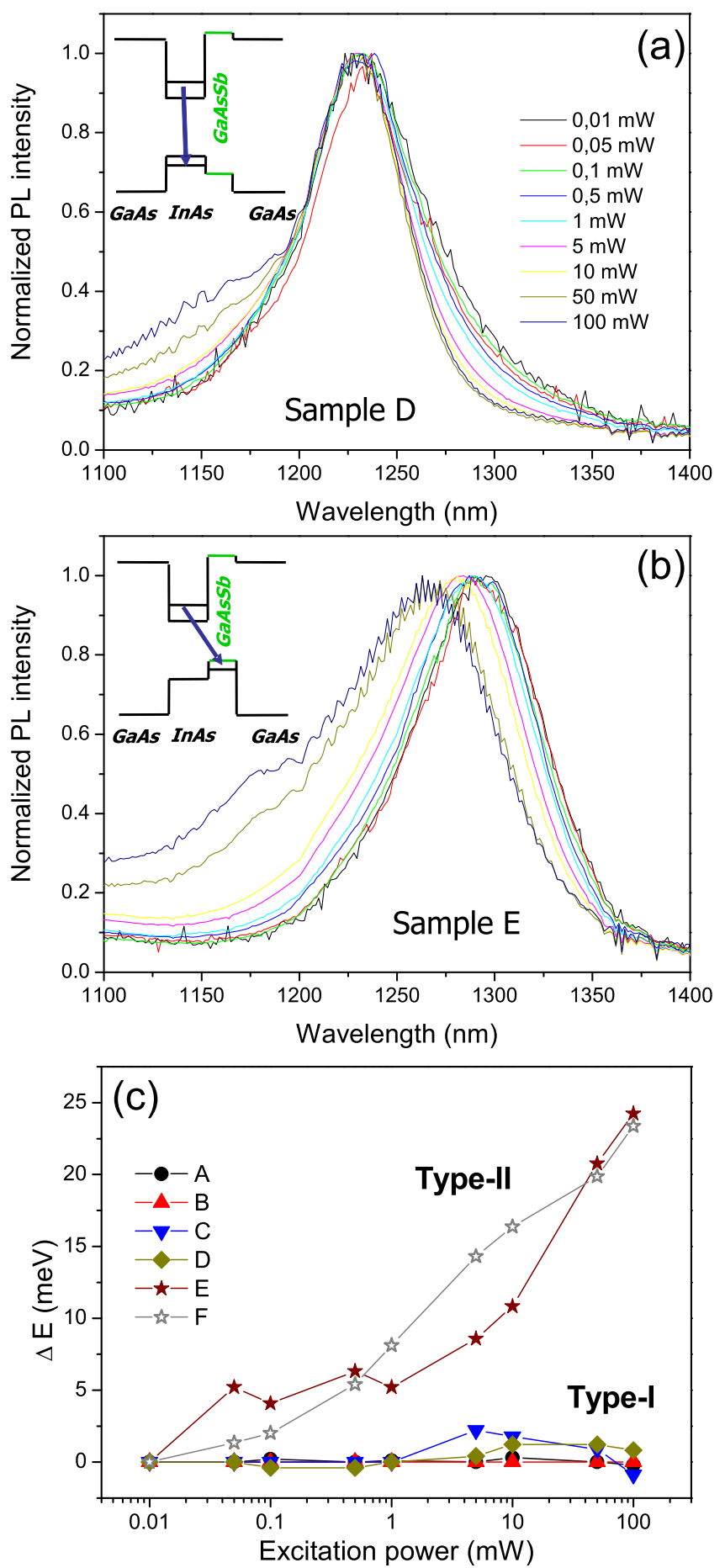

FIG. 4. Normalized PL spectra measured at $15 \mathrm{~K}$ as a function of excitation power for sample D (a) and sample E (b). The inset shows schematically the expected band alignment in each case. (c) Relative blue shift of the PL peak energy with excitation power for samples A to $F$. 
$\mathrm{Sb}$ content samples, i.e., the emission from the first excited state appears at much lower excitation powers than in type-I samples. Indeed, excited states are typically observed at lower carrier densities in type-II samples due to the faster saturation of the ground state because of the longer carrier lifetime due to the spatial separation. ${ }^{13}$ Therefore, the transition from a type-I to a type-II band alignment is likely taking place very close to sample $\mathrm{D}$, which corresponds to an $\mathrm{Sb}$ content of $\sim 16 \%$ (in good agreement with the values of 14-17\% indicated in Ref. 4 and 17\% in Ref. 26).

The transition to a type-II alignment could, in principle, explain the reduction of the PL integrated intensity at high $\mathrm{Sb}$ contents (see inset in Fig. 3). It must be noticed that, contrary to what happens at room temperature, ${ }^{18}$ the emission intensity of the type-II samples is considerably lower than that of the GaAs-capped QDs that act as the reference sample. Moreover, there is only a small initial improvement of the PL at moderate $\mathrm{Sb}$ contents (integrated intensity increases a factor of 1.3 from sample A $(0 \% \mathrm{Sb})$ to $\mathrm{C}(12 \%$ $\mathrm{Sb})$ ), while the increase in the same range of $\mathrm{Sb}$ is larger than one order of magnitude at room temperature. The improvement observed at room temperature was recently attributed to the gradual increase in QD height observed for $\mathrm{Sb}$ contents in that range, which should improve carrier confinement and electron-hole wavefunction overlap. ${ }^{18}$ The impact of the increased QD height on the PL will be theoretically analyzed in Sec. IV, by comparing the calculated oscillator strength for the ground transition. The mentioned differences observed between $15 \mathrm{~K}$ and room temperature point out to a strongly modified influence of the temperature on the PL emission of these structures in the presence of Sb.

In order to investigate this effect, the PL spectrum as a function of temperature was measured for samples A-F between $15 \mathrm{~K}$ and $340 \mathrm{~K}$. While no PL emission was observed in sample A above $315 \mathrm{~K}$, an intense spectrum was still obtained for samples B-F at $340 \mathrm{~K}$. The quenching of the PL intensity with temperature was found to be significantly smaller in the Sb-containing samples. Indeed, the integrated intensity decreases by a factor of 70 between $15 \mathrm{~K}$ and room temperature in sample A, while the decrease factor is only 7 in sample C. If the ratio between the integrated intensity at $15 \mathrm{~K}$ and room temperature is plotted as a function of the Sb content for all the samples (A-F), it is clear that there is a very strong initial reduction of the PL quenching with temperature when the amount of $\mathrm{Sb}$ in the capping layer increases (Fig. 5). For Sb contents above $\sim 12 \% \mathrm{Sb}$, the magnitude of the quenching remains approximately constant. The enhanced PL thermal stability in the presence of Sb represents an important advantage regarding the applications since it would improve the temperature performance of long wavelength devices.

These results demonstrate that the evolution of the PL with temperature is strongly modified by adding $\mathrm{Sb}$ to the capping layer. The reduced quenching with temperature in the presence of $\mathrm{Sb}$ gives rise to an improved PL emission at room temperature compared to standard InAs/GaAs QDs even in the type-II samples, contrary to what is observed at low temperatures. This is clear from the inset table in Fig. 5, in which the PL integrated intensity of samples $\mathrm{C}$ and $\mathrm{F}$

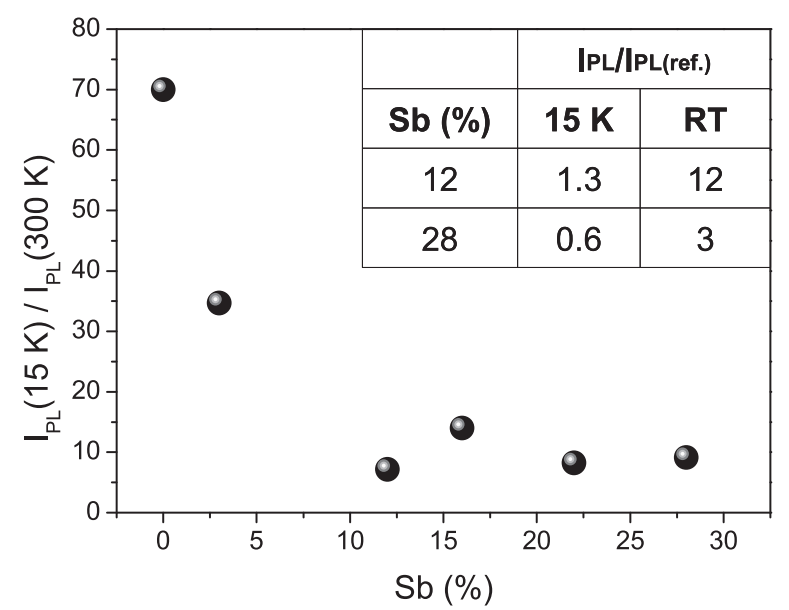

FIG. 5. Ratio between the PL integrated intensity at $15 \mathrm{~K}$ and $300 \mathrm{~K}$ as a function of the $\mathrm{Sb}$ content. The inset table shows the PL integrated intensity of samples $\mathrm{C}$ and $\mathrm{F}$ normalized to that of the reference (sample A) at $15 \mathrm{~K}$ and room temperature. A value of 1 means that the PL intensity is equal to that of the InAs/GaAs QDs.

normalized to that of the reference (sample A) is shown at $15 \mathrm{~K}$ and room temperature. A value of one means that the PL intensity is equal to that of the standard InAs/GaAs QDs. These two samples, C and F, are representative of what happens for the type-I and type-II samples, respectively. In the type-I sample C $(12 \% \mathrm{Sb})$, the PL gets enhanced by more than one order of magnitude at room temperature. In the case of the type-II sample $\mathrm{F}(28 \% \mathrm{Sb})$, there is an inversion in the integrated intensity ratio from $15 \mathrm{~K}$ to room temperature, yielding a more efficient luminescence than standard InAs/ GaAs QDs at room temperature. The fact that the integrated intensity of the indirect transition-based PL of the type-II samples can be much higher than that of the type-I reference is a very surprising fact which has not been satisfactorily explained yet. We will try to give some insight on this issue in Sec. IV.

In order to extract the thermal activation energies for PL quenching, a linear fit is performed to the Arrhenius plot of the PL integrated intensity as a function of the inverse temperature in the strong quenching regime (Fig. 6). The activation energies are obtained by fitting 7-8 points in the temperature range from 285 to $340 \mathrm{~K}$, in order to ensure a high linear correlation coefficient $>0.9$. The obtained activation energies are shown in the inset of Fig. 6. The activation energy in samples B-D (type-I alignment) is higher than in the reference (A). Nevertheless, and in spite of the reduced quenching, the activation energy is significantly smaller in the high Sb content type-II samples E and F. This behavior is different to what could be in principle expected and what is actually found in the case of InAs QDs capped with $\mathrm{GaSb}^{7}$ or InGaAsSb ${ }^{27}$ where a reduced PL quenching is linked to a higher activation energy. The carrier escape mechanisms giving rise to the observed activation energies will be discussed in Sec. IV.

\section{Electronic structure calculation}

In an effort to understand the observed results, the NEXTNANO ++ package has been used for calculating the 


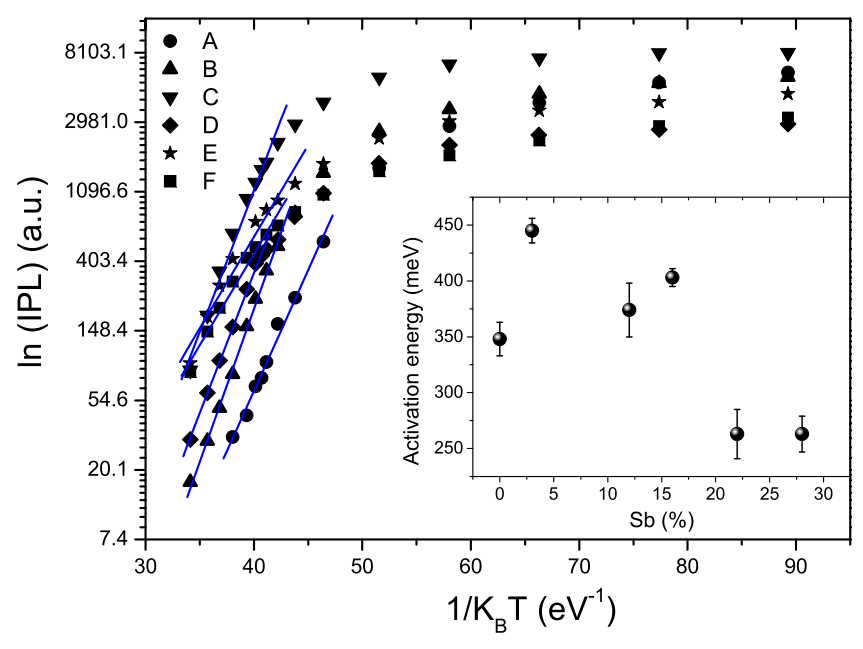

FIG. 6. (a) Arrhenius plot of the PL integrated intensity versus the inverse of the temperature for samples A to $\mathrm{F}$ with a linear fit in the strong quenching regime. The inset shows the activation energies deduced from the fit as a function of the $\mathrm{Sb}$ content.

electronic structure of the QDs. ${ }^{28}$ The theoretical model it implements relies on using an $8 \times 8 \mathrm{k} \cdot \mathrm{p}$ model method. The QD model takes into account the impact on the electronic structure of the strain distribution resulting of the different lattice constants of the materials involved in the formation of the nanostructure and its associated linear piezoelectric field. The Schrödinger equation was solved in a box extending $10 \mathrm{~nm}$ from lateral and vertical limits of the QD. The continuum elastic and Poisson equations were solved inside a box extending $50 \mathrm{~nm}$ away from the QD. In all cases, Dirichlet boundary conditions were assumed. The band parameters were taken from Ref. 29. A realistic structure was simulated, in which the input QD size, shape, and composition, as well as the capping layer thickness and Sb content were obtained from the X-STM measurements. A comparison of an atomically resolved high resolution X-STM image of a QD with the structure considered in the calculations is shown in Fig. 7. The QDs were found to have an ellipsoidal shape similar to that already reported for InAs/GaAs QDs, ${ }^{30}$ with a constant diameter of $25 \mathrm{~nm}$. The QD height (h) was found to progressively increase with the $\mathrm{Sb}$ content from $3.3(0 \% \mathrm{Sb})$ to $7.5 \mathrm{~nm}(14 \% \mathrm{Sb})$, and then remains constant, as reported in Ref. 18. The composition of the QDs was estimated from images in which a local mean equalization filter was applied in order to enhance the atomic details (Fig. 7(a)). Due to the different size and bonding configurations, In and $\mathrm{Sb}$ atoms appear brighter than $\mathrm{Ga}$ and As atoms, respectively. There- fore, the dark spots reflect the presence of Ga atoms replacing In atoms inside the QDs. Although group III elements are not directly observable at negative voltage, the dark features correspond to As atoms affected by the distortion that $\mathrm{Ga}$ atoms induce in their surroundings. By counting the dark spots, an average Ga content inside the QDs of $25 \pm 10 \%$ was estimated. Due to the difficulty of distinguishing individual atomic features when their concentration is relatively high $(\sim 25 \%)$, the error of this method is large.

The wetting layer (WL) was considered as a very thin InAs quantum well of $0.5 \mathrm{~nm}$, in order to approximately match the total amount of InAs of 1.5 MLs measured by XSTM in the reference sample. With the size, shape, and composition derived from X-STM, the calculated energy for the ground transition in the reference sample $(0 \% \mathrm{Sb})$ at $15 \mathrm{~K}$ was $1121 \mathrm{meV}$, which resulted slightly smaller than the measured value of $1168 \mathrm{meV}$. A perfect match between the model and the experiment for the ground transition energy was found for a $33 \%$ Ga content, which is within the uncertainty of the measurement, and therefore this is the value used for the calculations. We must note however that the coulomb interaction between the electron and hole wavefunction has not been included in the calculation, which means that our the theoretical results present an error not smaller than the exciton binding energy. Klenovsky et al. reported an exciton binding energy for a similar system of 26 (12) $\mathrm{meV}$ for an $\mathrm{Sb}$ content in the capping layer of 10 (22) $\%{ }^{26}$ The obtained conduction and heavy hole band edge profiles along the growth direction (z) together with the ground energy level for electrons and holes are shown in Figs. 8(a)-8(c) for the models corresponding to samples with 0,12 , and $22 \%$ Sb. Figures $8(\mathrm{~d})-8(\mathrm{f})$ show the ground state hole probability density in the (1-10) plane for the different $\mathrm{Sb}$ contents. The model predicts the transition to a type-II alignment to take place for $16 \% \mathrm{Sb}$, in very good agreement with the value found experimentally. For Sb contents above $16 \%$, the hole wavefunction is located in the capping layer towards the base of the QD in the [110] direction, as shown in Fig. 8(f) (a similar behavior was recently predicted for similar structures in which the QD was considered to have a square base and a non-homogeneous In distribution). ${ }^{26}$ The inhomogeneous strain pulls the hole wavefunction towards the QD rim at its base, while the piezoelectric field squeezes the wave function along the [110] direction. This was observed through independent calculations where the piezoelectric effect was neglected (not shown). Therefore, the heavy hole band edge profile across the center of the QD
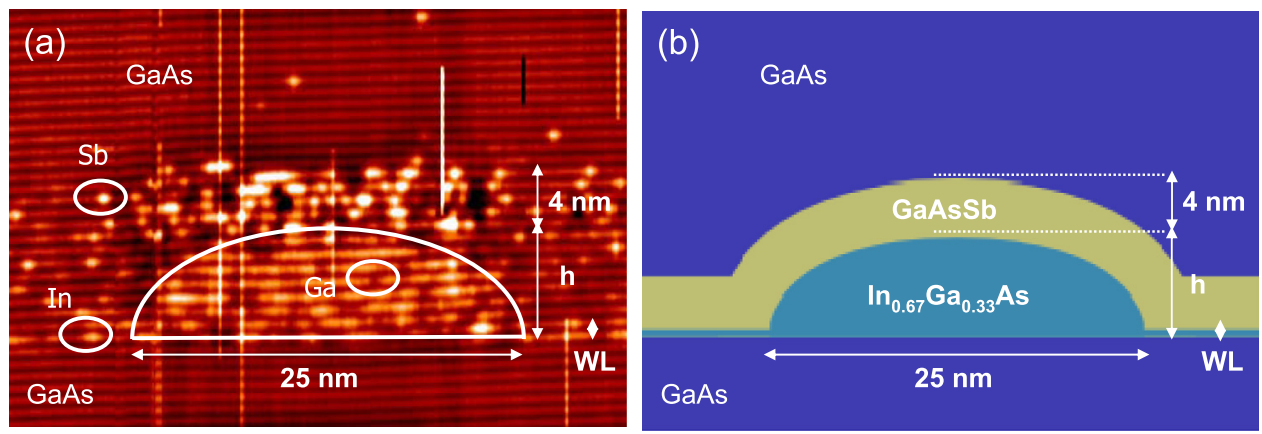

FIG. 7. (a) High resolution local mean equalization filtered image of a QD in LD $(22 \% \mathrm{Sb})$. The brighter spots correspond to $\mathrm{Sb}$ atoms in the As matrix and the dark features inside the QD indicate the presence of Ga. (b) Sketch of the QD structure considered in the calculations. The QD height (h) increases with the Sb content up to $16 \% \mathrm{Sb}$ and then remains constant: $3.3,4.2,5.2,6.7,7.5,7.5$, $7.5 \mathrm{~nm}$ for $0,3,7,11,16,22,28 \% \mathrm{Sb}$, respectively. 

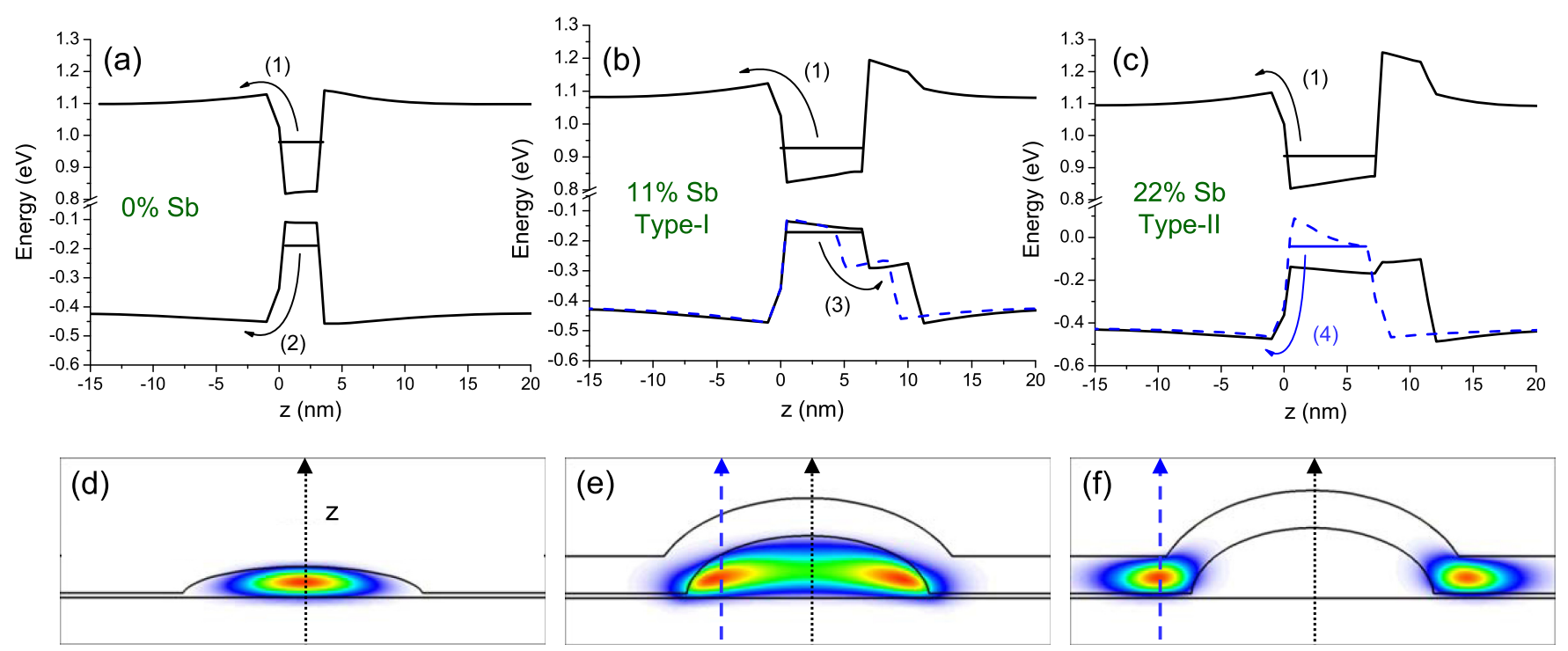

FIG. 8. (a)-(c) Calculated conduction and valence band edge profiles in the growth direction (z) across the center of the QD (black continuous lines), together with the energy of the electron and hole ground states for samples A, C, and E. In the case of samples C and E, the valence band edge profile is plotted also across a line close to the side of the QD base (blue dashed line). (d) to (f) show the distribution probability of the ground state hole wavefunction in the (1-10) plane.

does not suffice for describing the confinement potential exerted by the QD on the hole states, more notably for high Sb contents (black line in Fig. 8(c)). Hence, holes should not be in principle located on top of the QDs, as has been suggested. ${ }^{11}$ The transition to a type-II alignment can be considered to take place well before the valence band edge alignment between the QD and the capping layer on top is inverted. The potential profile for holes should therefore be plotted along a line close to the side of the base of the QD (dashed blue line in Figs. 8(c) and 8(f)). It is clear from the image that the delocalization of the heavy hole wavefunction towards the capping layer takes place progressively with the amount of $\mathrm{Sb}$. The consequence is that, even in the type-I samples in which the holes are located inside the $\mathrm{QD}$, the maximum of the probability density of the hole wavefunction is split in two parts located close to the QDcapping layer interface in the [110] direction (Fig. 8(e)).

Fig. 9 shows the calculated oscillator strength between electron and hole ground states normalized to that of InAs/ GaAs QDs as a function of the Sb content in the capping layer. The oscillator strength is defined as follows: ${ }^{31}$

$$
f=\frac{2}{m_{0} E_{\mathrm{eh}}}\left|\left\langle\Psi_{\mathrm{e}}|\hat{\mathrm{e}} \mathrm{p}| \Psi_{\mathrm{h}}\right\rangle\right|^{2},
$$

where $m_{0}$ is the electron mass, $E_{\text {eh }}$ is the electron-hole transition energy, $\Psi_{\mathrm{e}}\left(\Psi_{\mathrm{h}}\right)$ is the wavefunction of the electron (hole) ground state, $p$ is the momentum operator, and ê is a unit vector denoting the polarization of the absorbed light. Without loss of generality, we have only considered in the present study circularly polarized light.

\section{DISCUSSION}

Most of the optical properties highlighted in Sec. III can be explained with the aid of the theoretical study described above. The oscillator strength, inversely proportional to radiative lifetime, should in principle be related to the observed PL integrated intensity. Fig. 9 shows the oscillator strength trend corresponding to the simulations of the samples with different Sb contents. For Sb contents up to 16\% (transition to type-II alignment), there is a competition between the increased QD height and reduced strain on one side, which increase carrier localization and electron-hole wavefunction overlap, and the reduced QD-capping layer valence band offset on the other, which reduces hole localization, extending the hole wavefunction into the GaAsSb layer (see Fig. 8(e)). The model predicts that the increased QD height dominates only at low Sb contents below $\sim 7 \%$, where a small increase in the oscillator strength compared to standard InAs/GaAs QDs is predicted. In that range of Sb contents, the increase in QD height (from 3.3 to $5.25 \mathrm{~nm}$ ) has a stronger impact on the energy levels. ${ }^{32,33}$ Nevertheless, the

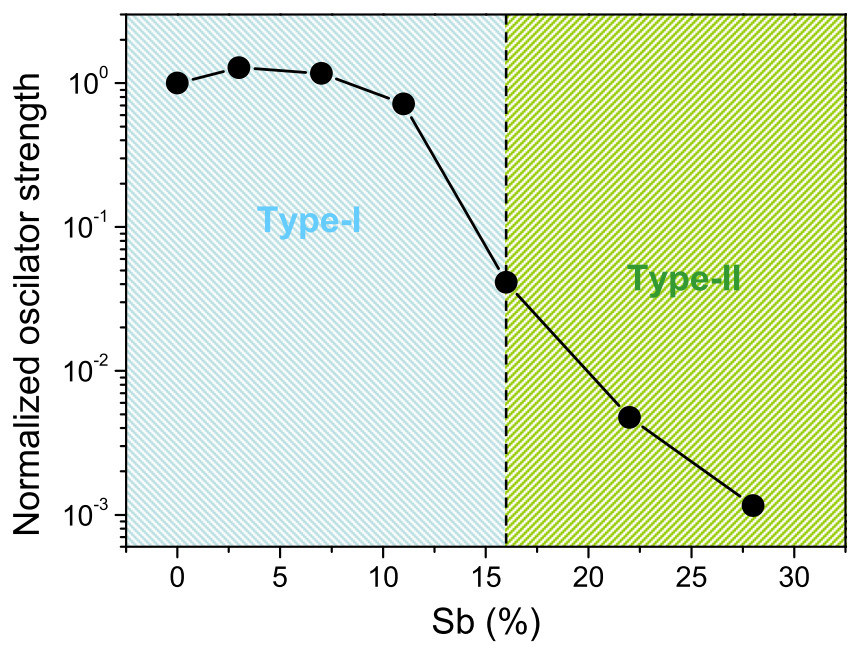

FIG. 9. Calculated oscillator strength between electron and heavy hole ground states as a function of the $\mathrm{Sb}$ content. The values are normalized to that of the reference sample $(0 \% \mathrm{Sb})$. The different background highlights the type-I and type-II regions. 
oscillator strength starts to decrease slowly with $\mathrm{Sb}$ above $3 \%$. For $\mathrm{Sb}$ contents above $16 \%$, the decrease is very strong due to the fact that the ground state hole wavefunction is localized outside the QD in the capping layer. It must be noticed that the oscillator strength is predicted to be three orders of magnitude smaller in the type-II sample with $28 \%$ $\mathrm{Sb}$ than in the GaAs-capped reference sample. This should in principle result in a strongly reduced PL signal.

If the oscillator strength is compared to the $15 \mathrm{~K} \mathrm{PL}$ integrated intensity (inset of Fig. 3), some important differences are observed: The integrated intensity increases up to $12 \% \mathrm{Sb}$, and it is only a factor of $\sim 2$ smaller in the $28 \% \mathrm{Sb}$ sample than in the reference, very far from the reduction of three orders of magnitude predicted for the oscillator strength (Fig. 9). It is true that if non-radiative lifetime is in any case much longer than radiative lifetime, the variations in radiative lifetime will not affect the efficiency so much. That could be the case at low temperature. Nevertheless, the unexpected high efficiency of the type-II samples compared to type-I is enhanced at room temperature (see table in Fig. 5), where non-radiative lifetime should be much shorter and comparable to radiative lifetime (PL intensity decreases 1 order of magnitude from $15 \mathrm{~K}$ to RT). In that situation, efficiency would be very strongly affected by the radiative lifetime unless we assume a huge increase in non-radiative lifetime in type-II samples of 3 orders of magnitude, which is not at all realistic. This points out to the fact that the PL efficiency is not dominated by the oscillator strength but by carrier dynamics, which are altered by the GaAsSb layer. One contribution to the observed differences between the oscillator strength and the PL integrated intensity could come from an increased carrier injection efficiency in the Sb-containing samples. Taking into account the QD density of $\sim 2 \times 10^{10} \mathrm{~cm}^{-2}$ and their size, their effective capture cross-section is only $9 \%$ of that of the WL. Therefore, carriers are mostly injected in InAs/GaAs QDs through the WL, which is indeed traditionally assumed to have a key role in the QD carrier relaxation. ${ }^{34,35}$ If the GaAsSb capping layer is added, holes could be trapped there before relaxing into the $\mathrm{WL}$, and would be injected trough that layer, giving rise to a separate electron and hole injection. Indeed, the electrons photoexcited in the GaAs capping located above the QDs will be backscattered by the capping layer for Sb concentrations greater than a threshold value. As illustrated in Figs. 8(b) and 8(c), the capping layer acts as a barrier for electrons. The separate injection assumes that carriers are behaving as uncorrelated electron-hole pairs, as it was previously proposed for InAs/GaAs QDs by Le Ru et al. ${ }^{34}$

The influence of carrier dynamics would become even more important at room temperature, with carriers having more energy to escape from traps or clusters in the WL and capping layer or from the QDs. Indeed, the difference between the oscillator strength and the PL integrated intensity is more noticeable at room temperature, where the integrated intensity is improved by one order of magnitude for $12 \% \mathrm{Sb}$ and the type-II samples show a much stronger PL signal than the standard InAs/GaAs QDs (see table in Fig. 5). This cannot be explained by the predicted oscillator strength and suggests again that the increased confinement

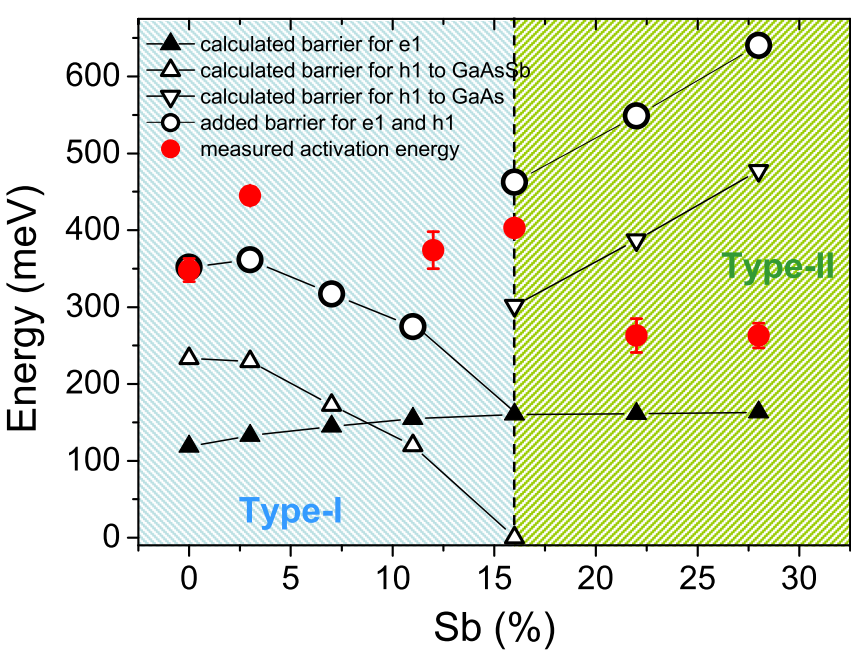

FIG. 10. Measured PL activation energy together with the calculated energy barriers for the electron ground state, the heavy hole ground state, and the addition of the calculated barriers for electron and hole ground states (added barrier for e1 and h1), as a function of the Sb content. The different background highlights the type-I and type-II regions.

induced by the increased QD height and reduced strain is not the main factor explaining the PL improvement.

The different energy barriers preventing electrons and holes to escape from the QDs before radiative recombination takes place can be extracted from the modeled band structure, as indicated in Fig. 8. The possible carrier escape mechanisms in the three different representative situations are schematically shown in the figure: electron escape to the GaAs barrier (1), hole escape from the QD to the GaAs barrier (2), hole escape from the QD to the GaAsSb capping layer (3), and hole escape from the GaAsSb capping layer to the GaAs barrier (4). The calculated barrier energy for electrons in the ground state increases slightly with the Sb content (Fig. 10), due to the decrease in the confinement energy resulting from the progressively increase in the QD height and reduced strain. These two factors also reduce the confinement energy of the hole ground state, but in this case, they compete with the reduced QD-capping layer valence band offset. The result is that the barrier energy for the hole ground state to the GaAsSb layer (escape mechanism (3)) remains almost constant for very low Sb contents $(\sim 3 \%)$ and then decreases for Sb contents up to $16 \%$ (Fig. 10). This energy becomes eventually zero at $16 \% \mathrm{Sb}$, when entering into the type-II region, because the hole ground state is located outside the QD in the GaAsSb capping layer (Figs. 8(c) and 8(f)). In this case, the holes would have to escape to the GaAs barrier (escape mechanism (4)), and therefore the barrier for hole escape is strongly increased (Fig. 10).

If the barriers for electrons and holes are added, the total barrier height agrees very well with the measured activation energy in the reference sample (see Fig. 10). In the case of the type-I samples, the activation energy seems to follow also the trend of the calculated total barrier height, with a small decrease from 3 to $12 \%$ and an increase for $16 \%$ (in the border of the two alignment regions). Nevertheless, the measured activation energies do not fit so well with the calculations in this case in terms of absolute values. Taking into 
account the complexity of the Sb-containing structures and the number of parameters involved in the model, the difference is reasonable and the calculations can mainly be used to discriminate between different escape mechanisms. From this comparison, it can be concluded that escape of both electrons and holes is most likely the main PL quenching mechanism for $\mathrm{Sb}$ contents up to $16 \%$ (in the type-I region), and that they do not behave as excitons or correlated pairs (if that was the case, the measured activation energy should be half of the total barrier height). ${ }^{36}$ Nevertheless, there is a difference between the case of $0 \% \mathrm{Sb}$ and the $\mathrm{Sb}$ containing samples: When $\mathrm{Sb}$ is present, the holes escape to the thin GaAsSb layer, where they remain confined and redistribution and retraping processes between QDs could take place, giving rise to a reduced effective hole escape rate. This could be especially important if the holes are the fastest escaping carriers, as could be the case for $\mathrm{Sb}$ contents between 8 and $16 \%$, in which the barrier energy for holes is considerably smaller than for electrons (see Fig. 10). The retraping process could explain the reduced PL quenching with temperature in that range (a reduced dependence of the PL with temperature due to retraping processes in $\mathrm{InAs} / \mathrm{GaAs}$ QDs has been already observed). ${ }^{37}$

For $\mathrm{Sb}$ contents above $16 \%$ (in the type-II region), the measured activation energy is much smaller than the total barrier energy (see Fig. 10). The measured values and the tendency with the $\mathrm{Sb}$ content fit best with the calculated barrier for electrons. Therefore, we suggest that, in this case, hole escape is suppressed and the main PL quenching mechanism with temperature is electron escape. This indicates again an independent behavior of electrons and holes, with only one type of carrier escaping. The altered carrier escape dynamics could partially explain the improved room temperature PL efficiency observed in the high Sb content samples.

\section{CONCLUSIONS}

The modified optical properties of InAs/GaAs QDs capped with a thin $\mathrm{GaAs}_{1-\mathrm{x}} \mathrm{Sb}_{\mathrm{x}}$ layer were analyzed by comparing to the band structure calculated with an $8 \times 8 \mathrm{k} \cdot \mathrm{p}$ model in which a realistic structure obtained from X-STM measurements was used. Despite the increased QD height and reduced strain when adding $\mathrm{Sb}$, which increase carrier confinement, the progressive hole wavefunction delocalization dominates the oscillator strength between electron and hole ground states, which decreases above $7 \% \mathrm{Sb}$ and cannot explain the observed evolution of the PL intensity with $\mathrm{Sb}$. Instead, the PL efficiency seems to be dominated by the injection and escape carrier dynamics, which are altered by the presence of the GaAsSb capping layer. Increasing the amount of $\mathrm{Sb}$ in the capping layer enhances the PL thermal stability, leading to an increased room temperature PL intensity compared to $\mathrm{Sb}$-free InAs/GaAs QDs even in the type-II samples. In standard GaAs-capped QDs, escape of both electrons and holes to the GaAs barrier is the main PL quenching mechanism. Nevertheless, for small-moderate $\mathrm{Sb}(<16 \%)$ for which the type I band alignment is kept, holes escape to the GaAsSb capping layer, where redistribution and retraping processes can take place. In the high $\mathrm{Sb}$ content type-II structures $(>16 \%)$, holes are already located in the GaAsSb layer and electron escape to the GaAs barrier is the main PL quenching mechanism.

We think that the presented results demonstrate that GaAsSb-capped InAs QDs have a strong potential as long wavelength emitters. On one hand, they are a very good candidate for developing lasers at $1.3 \mu \mathrm{m}$. That wavelength can be achieved at room temperature with moderate $\mathrm{Sb}$ contents $(\sim 12 \%)$ and still a type-I alignment. Indeed, the PL emission is optimum for those $\mathrm{Sb}$ contents so improved lasing characteristics compared to standard InAs/GaAs QD lasers can be expected. On the other hand, lasing at $1.55 \mu \mathrm{m}$ would require higher $\mathrm{Sb}$ contents and a type-II alignment. In this case, achieving laser emission can be more delicate due to the band alignment. Nevertheless, the strongly enhanced thermal stability of those samples and the fact that carrier dynamics compensate for the low oscillator strength should help in achieving room temperature lasing.

\section{ACKNOWLEDGMENTS}

This work has been supported by the Comunidad de Madrid through the project "Q\&C Light" (P2009/ESP-1503) and "Nanophotonic devices for optical communications" (CCG10-UPM/TIC-4932), by the European Union through the SANDiE Network of Excellence (Contract No. NMP4CT-2004-500101), by STW-VICI Grant No. 6631 and by contracts TEC2008-06756-C03-01 and TEC2011-29120C05-04. J. M. Ulloa would like to thank the support of the Spanish "Ministerio de Ciencia e Innovación" through the "Ramón y Cajal" program.

${ }^{1}$ K. Akahane, N. Yamamoto, and N. Ohtani, Physica E (Amsterdam) 21, 295 (2004).

${ }^{2}$ H. Y. Liu, M. J. Steer, T. J. Badcock, D. J. Mowbray, M. S. Skolnick, P. Navaretti, K. M. Groom, M. Hopkinson, and R. A. Hogg, Appl. Phys. Lett. 86, 143108 (2005).

${ }^{3}$ J. M. Ripalda, D. Granados, Y. González, A. M. Sánchez, S. I. Molina, and J. M. García, Appl. Phys. Lett. 87, 202108 (2005).

${ }^{4}$ H. Y. Liu, M. J. Steer, T. J. Badcock, D. J. Mowbray, M. S. Skolnick, F. Suarez, J. S. Ng, M. Hopkinson, and J. P. R. David, J. Appl. Phys. 99, 046104 (2006).

${ }^{5}$ T. Matsuura, T. Miyamoto, M. Ohta, and F. Koyama, Phys. Status Solidi C 3, 516 (2006).

${ }^{6}$ D. Guimard, S. Tsukamoto, M. Nishioka, and Y. Arakawa, Appl. Phys. Lett. 89, 083116 (2006).

${ }^{7}$ J. M. Ripalda, D. Alonso-Álvarez, B. Alén, A. G. Taboada, J. M. García, Y. González, and L. González, Appl. Phys. Lett. 91, 012111 (2007).

${ }^{8}$ J. M. Ulloa, I. W. D. Drouzas, P. M. Koenraad, D. J. Mowbray, M. J. Steer, H. Y. Liu, and M. Hopkinson, Appl. Phys. Lett. 90, 213105 (2007).

${ }^{9}$ S. I. Molina, A. M. Sánchez, A. M. Beltrán, D. L. Sales, T. Ben, M. F. Chisholm, M. Varela, S. J. Pennycook, P. L. Galindo, A. J. Papworth, P. J. Goodhew, and J. M. Ripalda, Appl. Phys. Lett. 91, 263105 (2007).

${ }^{10}$ T. T. Chen, C. L. Cheng, Y. F. Chen, F. Y. Chang, H. H. Lin, C.-T. Wu, and C.-H. Chen, Phys. Rev. B 75, 033310 (2007).

${ }^{11}$ C. Y. Jin, H. Y. Liu, S. Y. Zhang, Q. Jiang, S. L. Liew, M. Hopkinson, T. J. Badcock, E. Nabavi, and D. J. Mowbray, Appl. Phys. Lett. 91, 021102 (2007).

${ }^{12}$ J. M. Ulloa, C. Çelebi, P. M. Koenraad, A. Simon, E. Gapihan, A. Letoublon, N. Bertru, J. Drouzas, D. J. Mowbray, M. J. Steer, and M. Hopkinson, J. Appl. Phys. 101, 081707 (2007).

${ }^{13}$ Y. D. Jang, T. J. Badcock, D. J. Mowbray, M. S. Skolnick, J. Park, D. Lee, H. Y. Liu, M. J. Steer, and M. Hopkinson, Appl. Phys. Lett. 92, 251905 (2008).

${ }^{14}$ W.-H. Chang, Y.-A. Liao, W.-T. Hsu, M.-C. Lee, P.-C. Chiu, and J.-I. Chyi, Appl. Phys. Lett. 93, 033107 (2008). 
${ }^{15}$ V. Haxha, I. Drouzas, J. M. Ulloa, M. Bozkurt, P. M. Koenraad, D. J. Mowbray, H. Y. Liu, M. J. Steer, M. Hopkinson, and M. A. Migliorato, Phys. Rev. B 80, 165334 (2009).

${ }^{16}$ Y.-A. Liao, W.-T. Hsu, P.-C. Chiu, J.-I. Chyi, and W.-H. Chang, Appl. Phys. Lett. 94, 053101 (2009).

${ }^{17}$ D. Guimard, M. Ishida, L. Li, M. Nishioka, Y. Tanaka, H. Sudo, T. Yamamoto, H. Kondo, M. Sugawara, and Y. Arakawa, Appl. Phys. Lett. 94, 103116 (2009).

${ }^{18}$ J. M. Ulloa, R. Gargallo-Caballero, M. Bozkurt, M. del Moral, A. Guzmán, P. M. Koenraad, and A. Hierro, Phys. Rev. B 81, 165305 (2010).

${ }^{19}$ A. G. Taboada, A. M. Sánchez, A. M. Beltrán, M. Bozkurt, D. AlonsoÁlvarez, B. Alén, A. Rivera, J. M. Ripalda, J. M. Llorens, J. MartínSánchez, Y. González, J. M. Ulloa, J. M. García, S. I. Molina, and P. M. Koenraad, Phys. Rev. B 82, 235316 (2010).

${ }^{20}$ M. Montes Bajo, J. M. Ulloa, M. del Moral, A. Guzmán, and A. Hierro, IEEE J. Quantum Electron. 47, 1547 (2011).

${ }^{21}$ W.-T. Hsu, Y.-A. Liao, F.-C. Hsu, P.-C. Chiu, J.-I. Chyi, and W.-H. Chang, Appl. Phys. Lett. 99, 073108 (2011).

${ }^{22}$ J. M. Ulloa, D. F. Reyes, M. Montes, K. Yamamoto, D. L. Sales, D. Gonzalez, A. Guzman, and A. Hierro, Appl. Phys. Lett. 100, 013107 (2012).

${ }^{23}$ J. M. Ulloa, P. M. Koenraad, M. Bonnet-Eymard, A. Létoublon, and N. Bertru, J. Appl. Phys. 107, 074309 (2010).

${ }^{24}$ R. M. Feenstra, Physica B: Condensed Matter 273, 796 (1999).
${ }^{25}$ D. M. Bruls, J. W. A. M. Vugs, P. M. Koenraad, H. W. M. Salemink, J. H. Wolter, M. Hopkinson, M. S. Skolnick, F. Long, and S. P. A. Gill, Appl. Phys. Lett. 81, 1708 (2002).

${ }^{26}$ P. Klenovsky, V. Krapek, D. Munzar, and J. Humlicek, Appl. Phys. Lett. 97, 203107 (2010).

${ }^{27}$ W.-S. Liu, D. M. T. Kuo, J.-I. Chyi, W.-Y. Chen, H.-S. Chang, and T.-M. Hsu, Appl. Phys Lett. 89, 243103 (2006).

${ }^{28}$ S. Birner, T. Zibold, T. Andlauer, T. Kubis, M. Sabathil, A. Trellakis, and P. Vogl, IEEE Trans. Electron Devices 54, 2137 (2007).

${ }^{29}$ I. Vurgaftman, J. R. Meyer, and L. R. Ram-Mohan, J. Appl. Phys. 89, $5815(2001)$

${ }^{30}$ J. H. Blokland, M. Bozkurt, J. M. Ulloa, D. Reuter, A. D. Wieck, P. M. Koenraad, P. C. M. Christianen, and J. C. Maan, Appl. Phys Lett. 94, 023107 (2009).

${ }^{31}$ P. Y. Yu and M. Cardona, Fundamentals of Semiconductors (SpringerVerlag, 1999), p. 251.

${ }^{32}$ G. A. Narvaez, G. Bester, and A. Zunger, J. Appl. Phys. 98, 043708 (2005).

${ }^{33}$ H. Fu and A. Zunger, Phys. Rev. B 56, 1496 (1997).

${ }^{34}$ E. C. Le Ru, J. Fack, and R. Murray, Phys. Rev. B 67, 245318 (2003).

${ }^{35}$ D. Morris, N. Perret, and S. Fafard, Appl. Phys. Lett. 75, 3593 (1999).

${ }^{36}$ W. Yang, R. R. Lowe-Webb, H. Lee, and P. C. Sercel, Phys. Rev. B 56, 13314 (1997).

${ }^{37}$ S. Sanguinetti, M. Henini, M. Grassi Alessi, M. Capizzi, P. Frigeri, and S. Franchi, Phys. Rev. B 60, 8276 (1999). 